\title{
EL SISTEMA PRIVADO DE PENSIONES EN CHILE Y SUS RESGUARDOS CONSTITUCIONALES
}

\author{
THE CHILEAN PRIVATE PENSION SYSTEM AND ITS \\ CONSTITUTIONAL SAFEGUARDS
}

\section{Florencia Larraín VillanueVa*}

\section{INTRODUCCIÓN}

El 1 de mayo de este año se cumplieron 30 años de la entrada en vigencia del sistema privado de pensiones, una de las mayores reformas al rol del Estado en cuanto a la seguridad social y pionera en el mundo. Nacida bajo el régimen de Augusto Pinochet, el sistema se ha mantenido sin modificaciones sustanciales durante gobiernos de variadas ideologías y sesgos. Esta realidad contrasta con la de otros países de Latinoamérica que, tras algunos años de iniciado los cambios han efectuado reformas importantes e incluso nacionalizado de vuelta sus sistemas privados de pensiones. Es el caso de Argentina en 2009 y de Bolivia a fines del año pasado. ¿Qué variables servirían para explicar estos escenarios tan diversos? No cabe duda que el problema es complejo y las explicaciones variadas. Se relacionan con coyunturas políticas, con estilos de liderazgo, con escenarios económicos distintos y particularidades de cada cultura. Sin embargo, en el análisis institucional es donde mayores claves son posibles de encontrar para responder a esta interrogante.

El presente trabajo sostiene que la Reforma Previsional de 1980 no puede desli-

\footnotetext{
* Profesora de Derecho y de Latin American Politics, Universidad de los Andes. Email: floralarrain@yahoo.es. Licenciada en Ciencias Jurídicas y Sociales, Pontificia Universidad Católica de Chile; Master in European Political Studies, Universidad de Heidelberg.
}

garse del diseño institucional en la cual se inserta y solo con el marco constitucional que ampara y defiende sus pilares básicos puede entenderse su estabilidad en el tiempo. Estos últimos 30 años de historia chilena han visto pasar gobiernos de distintas ideologías y la transformación de muchas instituciones. En el ámbito previsional, no obstante haberse introducido múltiples y significativas reformas, el sistema ha presentado una marcada estabilidad. Considerando la magnitud de la reforma introducida al comienzo de los años 80 , las críticas al sistema, la cantidad de detractores del mismo y su marcada vinculación con los principios establecidos durante el Régimen Militar, su permanencia por más de 30 años merece ser analizada en detalle.

Este análisis busca entregar ciertas claves sobre el rol que las garantías y recursos establecidos en la Constitución de 1980 han jugado en la permanencia del sistema. Sostiene que, no obstante la existencia de una serie de otras variables -económicas, sociales, culturales, políticas- que puedan haber influido en su estabilidad, las variables institucionales tienen un potencial explicativo innegable para el análisis de un comportamiento tan paradójico: un sistema abiertamente resistido por una gran cantidad de agentes políticos que ha sido capaz de permanecer en el tiempo sin sufrir modificaciones sustanciales.

Antes de comenzar el análisis vale la pena detenerse y revisar algunos cuestionamientos. El primero de ellos sería ¿por qué 
considerar como algo anómalo o excepcional la estabilidad de una política pública, especialmente en el ámbito previsional? Tal como han señalado múltiples especialistas en el terreno de las políticas sociales y específicamente en el área de las pensiones, la norma es la estabilidad. El llamado "path dependency" surge como el principio que sirve como fundamento a la institucionalización y que en la práctica significa que una vez seguido un cierto camino, los costos de revertir esta decisión son cada vez más altos. William Sewell ${ }^{1}$ define el fenómeno como el efecto que producen los hechos anteriores en los posibles resultados de una secuencia de eventos que ocurre o se manifiestan en el futuro. En este contexto, es que podríamos afirmar que la estabilidad del sistema de pensiones chileno es una manifestación clara del principio de que la historia pesa y que los cambios radicales generalmente van asociados a costos muy altos al momento de revertirlos. Este principio sirve para justificar la resistencia al cambio que exhiben muchos países que experimentan hoy crisis de envejecimiento de su población y el consecuente aumento de sus gastos por concepto de previsión. Efectivamente, y respondiendo la pregunta recién planteada, la estabilidad del sistema chileno no debiera llamar la atención sino, más bien, debiera hacerlo la profundidad y el cambio de paradigma que implicó la introducción del sistema de pensiones. El presente análisis, sin embargo, considera la estabilidad como un fenómeno digno de ser analizado a la luz de los recientes cambios introducidos por países vecinos que, habiendo adoptado

\footnotetext{
${ }^{1}$ Véase Sewell, William (1996): "Three Temporalities: Toward an Eventful Sociology" en MC DONALD, Terrance (Ed): The Historic turn in Human Sciences (Ann Arbor, University of Michigan Press) pp. 262-263.
}

durante los años 90 (1994 en el caso de Argentina y 1997 en el de Bolivia) cambios en la línea de privatizar sus respectivos sistemas de pensiones, han modificado sus respectivas legislaciones para devolver al Estado su rol de principal agente en materia previsional. A la luz de estos hechos recientes es que cabría una segunda pregunta: ¿Sería posible en Chile adoptar cambios en esa línea, que significaran en la práctica una reforma sustancial al sistema de pensiones o su reemplazo por un sistema de reparto, como el existente hasta antes de 1980? Y como consecuencia de esta misma se deriva la pregunta central de este trabajo: ¿De qué forma ha influido la Constitución de 1980 en la estabilidad de los pilares fundamentales del sistema previsional chileno?

La forma en que se plantea esta última pregunta implica reconocer que en el transcurso de estos 30 años se han adoptado una serie de cambios al sistema que, no obstante su importancia, no pueden considerarse como modificaciones sustanciales a los pilares del sistema. Ellos han tenido funciones y lógicas bastante diversas, desde ampliar el espectro de instrumentos en los cuales pueden invertir las Administradoras de Fondos de Pensiones (AFP), la creación de los llamados multifondos, las modificaciones a los sistemas de rentas vitalicias, hasta el diseño y asignación de facultades a las instituciones encargadas de la supervisión y vigilancia del sistema. Cabe destacar dentro de ellas la reforma introducida durante el gobierno de Michelle Bachelet ${ }^{2}$ que implicó la creación de las llamadas pensiones solidarias, sistema complementario al establecido por el DL 3500 de 1980. Sin dejar de considerar el impacto de todas estas reformas, este análisis sostiene que a treinta años de

\footnotetext{
${ }^{2}$ Ley 20.255 .
} 
su creación, aún se mantienen los pilares fundamentales y los principios en los cuales se basa nuestro sistema previsional. Ello se relaciona con la institucionalidad en el cual se inserta el régimen previsional y con el orden y garantías establecidas en la Constitución Política de 1980.

El presente estudio se abocará primero a efectuar un breve recuento de la historia y puesta en marcha del sistema privado de pensiones y de su evolución hasta la fecha. Analizará la magnitud, impacto y características de las distintas modificaciones introducidas al mismo determinando en qué medida pueden considerarse como cambios sustanciales que afectan las bases del sistema. Luego examinará en detalle los preceptos, garantías, procedimientos y recursos constitucionales que pueden haber servido para preservar las bases del sistema de previsión. Finalmente, el estudio nos permitirá concluir en qué medida tales disposiciones actuaron como resguardo de la estabilidad del sistema en el tiempo.

\section{EL SISTEMA DE PENSIONES CHILENO Y SU EVOLUCIÓN EN EL TIEMPO}

El Sistema Privado de Pensiones introducido a partir de 1980 y la consiguiente puesta en marcha de las llamadas AFP, que hoy cumplen 30 años de existencia, marcan un quiebre en el paradigma existente hasta esa fecha. Chile, al igual que muchos otros países latinoamericanos, fue heredero de los fundamentos y las instituciones diseñadas en los tiempos de Bismarck en 1881 y profundizadas por Lord Beveridge en su conocido e influyente informe ${ }^{3}$. En respuesta a los

${ }^{3}$ Beveridge, William, 20 de noviembre de 1942, "Social Insurance and Allied Services". Para un análisis resumido sobre su contenido ver: Bowen Herrera, denominados "estados de necesidad" la sociedad se hacía cargo de los miembros que, por su edad o estado de salud, no podían contribuir, debiendo el Estado financiar tales beneficios con cargo a las contribuciones de la población laboral activa. Este sistema, el cual suponía la creación de un fondo único alimentado por los fondos aportados por el Estado, los trabajadores y los empleadores, fue el sistema predominante en los sistemas democráticos desde segunda mitad del siglo XX. En Chile el sistema se institucionalizó bajo la forma de "Cajas", que otorgaban prestaciones muy diversas y contemplaban requisitos diferentes para acceder a beneficios previsionales. Contrario a lo que podría imaginarse, el Servicio de Seguro Social, entidad que concentraba al mayor número de trabajadores que contaban con los menores ingresos, otorgaba menores beneficios e imponía las mayores dificultades para acceder a una jubilación. Esta enorme cantidad de sistemas previsionales fundado en una maraña de cuerpos jurídicos permitía que los grupos de trabajadores con mayor nivel de poder político consiguieran los mayores beneficios previsionales.

El desgaste que experimentó en el tiempo el sistema de reparto "a la chilena", su fuerte clientelismo y las críticas generalizadas hacia las discriminaciones e ineficiencias que conllevaba, pueden servir en parte para entender la estabilidad del actual sistema. Ya desde mediados del siglo $\mathrm{XX}$, presidentes de diversas inclinaciones políticas -Jorge Alessandri, Eduardo Frei Montalva y Salvador Allende- manifestaron sus críticas al sistema imperante y propusieron implementar cambios en la línea de contar con un sistema de previsión justo y

Alfredo (1992): Introducción a la Seguridad Social (Santiago, Editorial Jurídica de Chile) pp. 75-77. 
eficiente. Sin perjuicio de ello, la opción de reemplazar el sistema de reparto por uno de capitalización individual, no fue evaluada ni presentada como alternativa hasta el año $1974^{4}$. Las primeras ideas en este sentido se encuentran en informes elaborados por ODEPLAN bajo la dirección de Miguel Kast, y en un capítulo especial del Ladrillo5.

Con respecto a la gestación de este sistema, vale la pena consignar que, encontrándose el Congreso en receso a partir del 11 de septiembre de 1973, la discusión de la reforma no fue fruto de un debate parlamentario $^{6}$. La potestad legislativa se encontraba radicada en la Junta de Gobierno y específicamente la competencia en materias del área social, que incluía los Ministerios de Trabajo y Salud, correspondía a la $\mathrm{FACH}$, en cuyo interior no existía una visión favorable sobre este proyecto. Con la salida del General Leigh de la Junta de Gobierno en julio de 1978 se aprueban una serie de iniciativas legales que prepararon la vía a la reforma. Fue el Ministro del Trabajo, José Piñera, quien estuvo a cargo de materializarla, previa discusión y apro-

\footnotetext{
4 Para analizar algunos de estos aspectos, ver Godoy, Óscar/Valdés Salvador (1994): Democracia y Previsión en Chile: Experiencia con Dos Sistemas (Santiago, Cuadernos de Economía, Año 31, No 93) pp. $137-$ 143.

5 Para una versión completa de El Ladrillo: Bases de la Política Económica del Gobierno Militar Chileno, ver Capítulo 2: Políticas Económicas Específicas, Políticas de Previsión y Seguridad Social Pgs 120-136: http://www.cepchile.cl/dms/lang_1/cat_794_pag_1. html

${ }^{6}$ Mesa-Lago, Carmelo (1996): "Pension Reform in Latin America. Importance and Evaluation of Privatization Approaches", en Glade, William (Ed): Bigger Economies, Smaller Governments, Privatization in Latin America (Colorado, Westview Press) pp. 89-134. Mesa-Lago sostiene al respecto que la reforma se implantó bajo un régimen autoritario, lo que permitió la toma de complejas decisiones sin discusión pública y sin ponderar debidamente sus consecuencias.
}

bación por parte de la Junta de Gobierno. Aun cuando el Ministro del Trabajo logró el objetivo fundamental de reformar el sistema de pensiones, este proyecto fue examinado con recelo al interior de la Fuerzas Armadas, cuya previsión se mantuvo al margen del nuevo sistema de pensiones.

El Decreto Ley No 3.500 publicado en el Diario Oficial del 13 de noviembre de 1980 estableció en Chile el "Nuevo Sistema de Pensiones", rompiendo en forma radical con el predicamento existente hasta esa fecha e instaurando un sistema de capitalización individual. El nuevo sistema, que comenzó a operar el 1 de mayo de 1981 con una docena de AFPs, contempló la creación de cuentas individuales de propiedad de cada trabajador en la cual se acumularían todas las contribuciones generadas durante su vida laboral, las cuales serían administradas por sociedades anónimas especiales que tendrían como giro específico esta tarea. En ese sentido, el cambio de paradigma consistía en relacionar los beneficios que el sistema proporciona con el ahorro o esfuerzo que realice el trabajador durante su vida laboral activa. El sistema de reparto imperante hasta esa fecha, en cambio, endosaba a la generación siguiente el pago de las pensiones de los trabajadores retirados y disociaba muchas veces el aporte realizado al sistema del beneficio a percibir. Con respecto a los trabajadores que no lograran reunir el monto de cotizaciones necesarias para financiar una pensión mínima, el nuevo sistema imponía al Estado el deber de garantizar el pago de pensiones mínimas.

Las modificaciones al sistema no se hicieron esperar y fueron abundantes ${ }^{7}$, efec-

\footnotetext{
${ }^{7}$ A 1990 el Decreto Ley 3.5000 contaba con más de 20 modificaciones a su texto original: D.L. No 3.626 D. Of. 21/02/81, D.L. No 3.650 D. Of. 11/03/81, Ley No 18.072 D. Of. 01/12/81, Ley No 18.086 D. Of. 31/12/81, Ley No 18.137 D. Of. 05/07/82,
} 
tuadas varias bajo la vigencia del régimen de Augusto Pinochet, tuvieron por objeto principalmente flexibilizar las posibilidades de la participación de las AFP en la compra de acciones de sociedades anónimas y adecuar las posibilidades de inversión a nuevos instrumentos financieros. Esta reforma fue seguida por una serie de cambios efectuados por diversos gobiernos de la Concertación, tendientes a flexibilizar las inversiones autorizadas a las AFP. Tales cambios, lejos de erosionar las bases del sistema, fueron consolidándolo y permitiendo mayores niveles de libertad no solo en cuanto a las inversiones que podían efectuar las administradoras, sino al nivel de riesgo que los cotizantes podían asumir en sus propias carteras y a la entrada de nuevos actores a la administración de fondos de pensiones ${ }^{8}$. Dada su extensión, el presente estudio no profundizará en cada uno de estos cambios, sino que se centrará en determinar si los mismos afectaron las bases del sistema original impuesto en 1980.

El diseño básico del nuevo sistema contempla la existencia de tres pilares ${ }^{9}$. En efecto, desde sus inicios es posible distin-

Ley No 18.196 D. Of. 29/12/82, Ley No 18.208 D. Of. 26/01/83, Ley No 18.225 D. Of. 28/06/83, Ley No 18.379 D. Of. $04 / 01 / 85$, Ley No 18.398 D. Of. $24 / 01 / 85$, Ley No 18.420 D. Of. $05 / 07 / 85$, Ley No 18.481 D. Of. $24 / 12 / 85$, Ley No 18.482 D. Of. 28/12/85, Ley No 18.520 D. Of. 13/06/86, Ley No 18.646 D. Of. 29/08/87, Ley No 18.646 D. Of. 29/08/87, Ley No 18.681 D. Of. $31 / 12 / 87$, Ley No 18.717 D. Of. 28/05/88, Ley No 18.753 D. Of. $28 / 10 / 88$, Ley No 18.768 D. Of. 29/12/88, Ley No 18.798 D. Of. 23/05/89, Ley No 18.840 D. Of. 10/10/89, Ley No 18.964 D. Of. 10/03/90.

${ }^{8}$ Vale la pena destacar por su profundidad la reforma efectuada por la Ley 19.795 de 28 de febrero de 2002 que crea los llamados "multifondos" y permite el ingreso de los Bancos, Compañías de Seguros y Fondos Mutuos a la administración de fondos previsionales.

9 www.safp.cl/573/propertyvalue-1701.html contiene un diseño actualizado del sistema y consigna sus principales reformas. guir un pilar no contributivo o solidario, un pilar contributivo basado principalmente en la capitalización individual y un tercer pilar voluntario compuesto por distintas fórmulas de ahorro complementario destinadas a mejorar la pensión de vejez. Este esquema, recomendado especialmente por el Banco Mundial a partir de la década de los $90^{10}$, permite estructurar un sistema de pensiones con un componente fiscal importante focalizado especialmente en aquel segmento de la población que, por diversas circunstancias, no presenta aportes en el sistema contributivo o su densidad de cotizaciones es tan baja que no le permitiría financiar una pensión mínima o de subsistencia. Este pilar es básico y sustancial al nuevo sistema de pensiones y ha sido reforzado en el tiempo estableciendo correcciones basadas en la equidad del género o en la mejoría de quienes presentan períodos prolongados de cesantía o bajos aportes al sistema. El segundo pilar, denominado contributivo, contempla contribuciones definidas y prestaciones indefinidas. Su objetivo principal es otorgar a quienes han concluido su etapa laboral activa un estándar de vida que se acerque razonablemente al que se gozaban durante su vida activa. Dentro de este pilar se incluye también un pequeño porcentaje de adscritos al antiguo sistema de reparto. Por último, el tercer pilar contempla la existencia de un sistema de ahorro complementario para la vejez (APV y la denominada cuenta 2) al cual se agregó en marzo de 2008 un sistema de ahorro

\footnotetext{
10 Vale la pena destacar en ese sentido el completo estudio preparado por esta entidad y contenido en el conocido libro "Averting the Old Age Crisis: Policies to Protect the Old and Promote Growth" (1994); The International Bank for Reconstruction and Development/ The World Bank, Oxford University Press.
} 
previsional colectivo y se introdujo la figura del afiliado voluntario.

Las modificaciones legales que se refieren a la materia han avanzado en la profundización de estos pilares pero sin afectar su esencia. Vale la pena consignar como una de las mayores reformas al sistema la introducida por la Ley No 20.255 a los tres pilares que conforman el sistema de pensiones. Tal como se consigna en el sitio web de la Superintendencia de AFP, tales modificaciones tuvieron por objeto "lograr un sistema integrado y coordinado que asegure la protección social a cada uno de los ciudadanos del país". Vale decir, en su conjunto, las disposiciones que han reformado al sistema se han ido integrando al mismo sin cambiar el objetivo fundamental ni la lógica con que fueron diseñadas originalmente.

\section{EL SISTEMA DE PENSIONES EN EL CONTEXTO DE LA CONSTITUCIÓN DE 1980}

El nacimiento de la Constitución de 1980, aprobada por plebiscito el 11 de septiembre de ese año y claramente ligada al régimen de Augusto Pinochet, ha sido objeto de ácidas críticas y de variadas reformas a lo largo de las tres décadas de su vigencia. Sin perjuicio de ello, existe un amplio consenso sobre los beneficios del diseño constitucional para la gestación de las profundas modernizaciones de la economía chilena. La definición del rol del Estado como subsidiario a la iniciativa privada y los límites y recursos que limitan su actuar han sido claves para introducir cambios radicales en las bases de nuestra economía e indispensables para el diseño del actual sistema de pensiones. Un estudio profundo sobre el diseño constitucional, sus ventajas y problemas excede claramente un trabajo como el propuesto. Este capítulo se limitará a señalar algunos de los aspectos institucionales básicos y se centrará en los resguardos que la misma Constitución establece en el ámbito de la Seguridad Social y concretamente de la previsión.

Dentro de las bases de la institucionalidad constitucional ${ }^{11}$, merece destacarse el reconocimiento explícito a la libertad y la igualdad en dignidad y derechos de la cual son dotados los hombres, a la familia como núcleo fundamental de la sociedad, al amparo y garantía por parte del Estado de los grupos intermedios a través de los cuales se organiza y estructura la sociedad y su adecuada autonomía y a la definición misma del rol del Estado al servicio de la persona humana teniendo como finalidad la de promover el bien común. Todas estas disposiciones se interpretan en armonía con la debida delimitación de los poderes públicos, del principio de la legalidad al cual se someten todos los órganos del Estado y a las garantías y recursos que la Constitución establezca $^{12}$. No se trata simplemente de un conjunto de principios teóricos sino que se aplican en forma concreta a los contenidos y formalidades que cumple todo el ordenamiento jurídico.

Concretamente en el ámbito de la seguridad social y de la previsión es necesario destacar la garantía establecida en el Artículo 19 no 18 de la Carta Fundamental que consagra el derecho a la seguridad social estableciendo para la regulación de su ejerci-

\footnotetext{
11 Para un análisis más profundo sobre las bases de la institucionalidad y su importancia ver: Diez URzúA, Sergio (1999): Persona y Valores: Su Protección Constitucional (Santiago, Editorial Jurídica) pp. 39-94

12 Para un análisis sistemático sobre el contenido del Orden Público Económico Chileno y la Libertad Económica ver: Fermandois, Arturo (2001): Derecho Constitucional Económico (Santiago, Ediciones Universidad Católica de Chile) pp. 65-191.
} 
cio leyes de quórum calificado. Esta norma claramente ha actuado como un resguardo de la estabilidad del sistema toda vez que para cualquier modificación debe contar con la mayoría absoluta de los diputados y senadores en ejercicio. Al respecto, este mismo precepto aclara el rol del Estado sobre la materia estableciendo que la acción de este estará dirigida a garantizar el acceso de todos los habitantes al goce de las prestaciones básicas uniformes, sea que se otorguen a través de instituciones públicas o privadas. Ello no obsta al deber del Estado de supervigilar el adecuado ejercicio del derecho a la seguridad social. La norma también explicita que la ley podrá establecer cotizaciones obligatorias.

La Constitución ha reservado además una serie de materias de ley que corresponden a la iniciativa exclusiva del Presidente de la República, entre las cuales destacan los proyectos de ley que tengan relación con la administración financiera o presupuestaria del Estado ${ }^{13}$. La misma iniciativa es necesaria para proyectos legales que pretendan establecer o modificar las normas sobre seguridad social o que incidan en ella, tanto en el sector público o privado o que busquen fijar, modificar, conceder o aumentar remuneraciones, jubilaciones, pensiones, montepíos ${ }^{14}$. En estas materias el rol del Congreso Nacional queda limitado, pudiendo solamente aceptar, disminuir o rechazar los emolumentos, beneficio, gastos y demás iniciativas sobre la materia que proponga el Presidente de la República ${ }^{15}$. Esta prerrogativa del Presidente fue discutida recientemente y fallada por el Tribunal Constitucional. El centro de la discusión

\footnotetext{
${ }^{13}$ Art. 62 inciso tercero.

${ }^{14}$ Art. 62 no 4 y no 6.

${ }^{15}$ Art. 62 inciso final.
}

giraba en torno a una discrepancia entre los colegisladores respecto a lo preceptuado en el artículo 65 de la Carta Fundamental, referente a la legitimidad y extensión de la iniciativa exclusiva del Presidente de la República en materia de gastos originados en beneficios a funcionarios y trabajadores y de modificaciones a normas de seguridad social. Al mismo tiempo, planteaba una discrepancia respecto a las atribuciones que otorga la citada norma en esos tópicos al Congreso Nacional. Al respecto, el Tribunal determinó que "las normas constitucionales sobre iniciativa exclusiva del Presidente de la República en materias legislativas guardan estrecha relación con la forma como nuestra Carta Fundamental ha consagrado el régimen presidencial de Gobierno. Tales normas permiten resguardar, asimismo, las esferas de competencia que la Constitución ha reservado para el Presidente de la República"16. Tomando en cuenta este y una serie de otros argumentos, el Tribunal Constitucional acogió el requerimiento deducido por el Presidente, declarándose inconstitucional el inciso primero del artículo 197 bis, que el Senado intentaba incorporar al Código del Trabajo.

Cabe destacar también como resguardo constitucional al sistema de pensiones chileno la garantía establecida en el Art. 19 $n^{\circ} 24$ de la Constitución que garantiza el derecho de propiedad en sus diversas especies sobre toda clase de bienes corporales e incorporales. Solo la ley puede establecer el modo de adquirir la propiedad, de usar, gozar y disponer de ella y las limitaciones y obligaciones que deriven de su función social. Esta comprende cuanto exijan los intereses generales de la Nación, la seguridad nacional, la utilidad y la salubridad públicas

\footnotetext{
${ }^{16}$ Sentencia del Tribunal Constitucional 2025-11 de 20 de julio de 2011, p. 69.
} 
y la conservación del patrimonio ambiental. Se dispone expresamente que nadie, en caso alguno, puede ser privado de su propiedad, del bien que recae o de alguno de los atributos o facultades esenciales al dominio, sino en virtud de ley general o especial que autorice la expropiación por causa de utilidad pública o de interés nacional calificada por el legislador. Esta garantía guarda estrecha relación con el sistema de previsión establecido mediante el Decreto Ley 3500 toda vez que el sistema de capitalización individual supone el derecho de propiedad por parte del afiliado de su respectivo fondo de pensión. De este dominio, aun cuando se encuentra afecto a un fin específico y sujeto a una serie de condiciones legales para ejercer su derecho a pensionarse, es que nacen una serie de derechos para el afiliado, entre los cuales se cuenta el de transferir sus fondos a otra Administradora, el de elegir el nivel de riesgo que quiere asumir, el de decidir si quiere efectuar aportes voluntarios en forma individual o colectiva etc.

Además de las garantías explícitas antes mencionadas, el Art. 19 n²6 consagra la seguridad de que los preceptos legales que por mandato de la Constitución regulen o complementen las garantías que esta establece o que las limiten en los casos que esta autorice, no podrán afectar los derechos en su esencia, ni imponer condiciones, tributos o requisitos que impidan su libre ejercicio.

Finalmente y como complemento a las garantías y procedimientos indicados, merece especial atención el rol que le ha cabido al Tribunal Constitucional en la delimitación en la práctica de tales conceptos. A partir de estos preceptos el Tribunal Constitucional ha ido elaborando las bases teóricas relativas al Orden Público Económico, concepto que, a su vez, ha servido para dirimir una serie de conflictos de gran relevancia práctica ${ }^{17}$. En el ámbito de la seguridad social y de la previsión, el Tribunal Constitucional ha debido fallar sobre una diversidad de conflictos que afectan los derechos y garantías en el ámbito previsional tanto de los agentes económicos involucrados como de los titulares de tales derechos ${ }^{18}$. Vale la pena destacar al respecto la sentencia Rol No 334 de 21 de agosto de 2001 en relación al requerimiento formulado por diversos senadores para que se resuelva sobre la constitucionalidad de un proyecto de ley que intentaba modificar el Decreto Ley No 3500 estableciendo normas relativas al otorgamiento de pensiones bajo la modalidad de rentas vitalicias. En este caso el Tribunal sentó un precedente importante en materia del resguardo del derecho de propiedad y de la libertad de cada afiliado al sistema a elegir el sistema bajo el cual desea pensionarse. En dicho fallo, el Tribunal determinó ${ }^{19}$ que si bien es efectivo que la Constitución convoca al legislador para regular el ejercicio del derecho a la

\footnotetext{
${ }^{17}$ Ver al respecto Sentencia Rol No 78 de 20 de septiembre de 1989 sobre el Proyecto de Ley Orgánica Constitucional del Banco Central y Sentencia Rol No 207 de 1995 sobre el caso "Deuda Subordinada", las cuales definen el sentido y alcance del Orden Público Económico a la luz de la Constitución de 1980.

18 Ver, entre otras, Sentencias Rol No 1032 de 4 de marzo de 2008 sobre Pensiones Solidarias; Rol No 184 de 7 de marzo de 1994 sobre Proyecto que modifica Ley de Mercado de Valores, Administración de Fondos Mutuos, de Fondos de Inversión, AFPs y Compañías de Seguros; Rol No 295 de 28 de septiembre de 1999 sobre creación de Cuenta 2 y perfeccionamiento de los mecanismos de medición de la rentabilidad mínima; Rol No 321 de 24 de abril de 2001 sobre Proyecto de Seguro de Desempleo; Rol No 338 de 10 de octubre de 2001 que flexibiliza inversiones de Fondos Mutuos y Compañías de Seguros y Rol No 345 de 30 de enero de 2002 sobre inversiones de Fondos de Pensiones.

${ }^{19}$ Considerando No 25 Sentencia Rol 334 de 21 de agosto de 2001.
} 
seguridad social, no es menos cierto que tal convocatoria tiene la categórica limitación establecida en el artículo 19 no 26 . En ese fallo el Tribunal juzgó que la regulación que efectuaba el proyecto objeto de la controversia afectaban en su esencia "el derecho de propiedad de los afiliados sobre sus fondos previsionales pues los privan de uno de los atributos fundamentales del dominio, como es la facultad de disponer de su titular de cumplirse las exigencias legales".

\section{CONCLUSIONES}

El rol que la Constitución de 1980 ha jugado en la estabilidad del sistema previsional no es fácil de determinar. Su estabilidad es en gran medida el reflejo de un consenso mayoritario a nivel político sobre la conveniencia práctica que plantea para la economía y el bienestar social un sistema basado en el ahorro individual ${ }^{20}$, donde el Estado asume un rol de garante y fiscalizador del mismo. Esta estabilidad política y económica sirve también para explicar la permanencia de la Constitución misma, el respeto a las garantías que ella contiene y su fortalecimiento en el plano institucional. Dicha realidad contrasta con la de países vecinos que han decidido modificar sus marcos constitucionales o legales, incluyendo sus sistemas previsionales, como respuesta a

\footnotetext{
${ }^{20} \mathrm{Al}$ respecto, resulta interesante la opinión del Presidente de la Central Unitaria de Trabajadores, Sr. Arturo Martínez avalando los beneficios del sistema: "Considero que los trabajadores chilenos deberían estar orgullosos del sistema de AFP y los sectores más acomodados deberian rendirles un homenaje por la cantidad de fondos y recursos que integran día a día a la economia nacional (...), se cree que son las AFP las que en definitiva aportan, invierten; claro, ellas lo hacen, pero son fondos de los trabajadores, son fondos nuestros" (Seminario Internacional OIT "El Sistema de Pensiones en Chile en el Contexto Mundial y de América Latina. (2004). P. 152)
}

problemas económicos o de gobernabilidad política.

La Constitución de 1980 introduce normas y garantías que van insertas en una concepción doctrinaria que otorga al Hombre una primacía sobre el Estado. Esta concepción tiene como consecuencia práctica, el respeto a los derechos inherentes a la persona humana, anteriores y superiores a los del Estado, limitando el principio de la soberanía popular. Este marco teórico determina además como principio rector en el plano constitucional económico, el rol subsidiario del Estado. Conforme el mismo texto constitucional dispone, el Estado está al servicio de la persona humana y su finalidad es promover el bien común. Reconoce y ampara específicamente a los cuerpos intermedios a través de los cuales se organiza la sociedad y les garantiza la adecuada autonomía para cumplir sus propios fines específicos. Este reconocimiento es en parte una derivación de la libertad de asociación. Estas declaraciones valóricas son una novedad de la Constitución de 1980. No existían en la Constitución de 1925 disposiciones que explicitaran la finalidad del Estado ni su esencia, ella se limitaba a consagrar que la soberanía residía en la Nación, sin consagrar límites que protegieran a los ciudadanos de los dictados de una mayoría política. Por último, aunque no por ello menos importante, es conveniente destacar lo preceptuado en el primer artículo de la Constitución que consagra que las personas nacen libres e iguales en dignidad y derechos, norma de la cual nacen los principios de libertad y de igualdad que cualquier disposición jurídica debe transparentar. Las garantías que se analizaron en detalle en el capítulo anterior no son más que una derivación de esta regla y sirven para referir y explicitar en detalle la materialización 
misma de la libertad y la igualdad en planos más específicos de la convivencia social. En el caso del presente trabajo, la libertad y la igualdad resultan ser los ejes principales y probablemente, a nivel más valórico y fundamental, la mejor defensa de las bases mismas del sistema previsional. El sistema previsional chileno instaurado mediante el Decreto Ley 3.500 respeta y potencia tanto la libertad de los individuos para elegir a quienes administran sus fondos previsionales y garantizan la igualdad de trato de todos quienes se pensionen.

Tal como se indica en este trabajo, el sistema previsional previo al establecido en 1980 era discriminatorio, ineficiente y expropiatorio. Coexistían una serie de beneficios y privilegios asignados en función del poder de presión de distintos grupos de trabajadores y no existía relación entre los aportes al sistema y los beneficios que este entregaba. Es probable que el descrédito de este sistema de reparto "a la chilena" haya influido también en alguna medida a afianzar un sistema basado en la capitalización individual. Sin embargo, es importante afirmar que, un sistema que reprodujera el anterior en este aspecto no tendría cabida pues entraría en contradicción con lo que dispone el Artículo 19 no2 de la Constitución ${ }^{21}$.

Sin embargo, analizando los preceptos que contienen y regulan el derecho a la seguridad social es donde podemos encontrar con mayor claridad los resguardos constitucionales que pueden explicar cómo y por qué las bases del sistema han permanecido intactas. La exigencia de leyes de quórum calificado para regular el ejercicio de este derecho y la iniciativa exclusiva del Presidente para fijar, modificar, conceder o

\footnotetext{
${ }^{21}$ Esta garantía consagra la igualdad ante la ley y explicita que en Chile no hay persona ni grupo privilegiados.
}

aumentar jubilaciones, pensiones o beneficios así como para establecer o modificar las normas sobre seguridad social o las que incidan en ella han influido claramente en la elaboración de modificaciones de consenso, sometidas al estudio de comisiones técnicas sobre la materia. Es el caso de la modificación propiciada por el gobierno de la Presidenta Michelle Bachelet, la cual fue objeto de un amplio debate y estudio al interior de la comisión de 15 expertos creada al efecto y presidida por Mario Marcel22.

La forma en que se establece el derecho a la seguridad social bajo la Constitución supone que el rol del Estado consiste en garantizar prestaciones básicas uniformes, ya sea a través de instituciones públicas o privadas. Este reconocimiento a la intervención de la empresa privada en el ámbito previsional supone necesariamente el respeto fundamental a dos garantías básicas en el ámbito del orden público económico: la de desarrollar cualquier actividad económica que no sea contraria a la moral, al orden público o la seguridad nacional, respetando las normas legales que la regulen ${ }^{23}$ y la no discriminación arbitraria que deben dar el Estado y sus organismos en materia económica ${ }^{24}$. Plantea claramente una profundización del princi-

\footnotetext{
${ }^{22} \mathrm{El}$ informe evacuado por la denominada Comisión Marcel el 6 de julio de 2006 contenía un diagnóstico, indicaba las fortalezas y debilidades del sistema y contenía una serie de propuestas que fueron discutidas en el Parlamento y sirvieron finalmente para la discusión y aprobación de la Ley de Pensiones Solidarias publicada el 2008. Informe del Consejo Asesor Presidencial para la Reforma del Sistema Previsional se encuentra disponible en: http://www. bcn.cl/carpeta_temas_profundidad/temas_profundidad.2007-09-13.2330905950/ResumenEjecutivo\%20Informe\%20Marcel.pdf [Fecha de Consulta: 22-12-2011]

${ }^{23}$ Art. 19 no 21 Constitución Política de la República.

${ }^{24}$ Art. 19 no 22 Constitución Política de la República.
} 
pio de la libertad que reconoce y ampara la Constitución.

La protección que consagra la Constitución al derecho de propiedad y los recursos constitucionales para asegurar su debido respeto también merecen una consideración especial al momento de analizar la estabilidad del sistema. Por la vía de convertir al trabajador en el protagonista principal de su propia previsión, el sistema lo ha dotado con derechos de propiedad explícitos sobre los fondos acumulados. Como consecuencia de ello, cualquier precepto legal que por mandato de la Constitución pudiera regular o complementar o limitar esta u otra de las garantías antes indicadas no podrá afectar los derechos en su esencia, ni imponer condiciones, tributos o requisitos que impidan su libre ejercicio. Esta protección es fundamental para asegurar el respeto a las bases mismas del sistema y ha sido reafirmada en los fallos del Tribunal Constitucional en los casos concretos en que los límites de tales garantías y la esfera de competencia de la iniciativa presidencial en esta área ha sido objeto de controversia. El aporte del Tribunal Constitucional en esta materia ha sido fundamental en el establecimiento, la definición y la profundización de estos derechos y puede ser considerado como un factor institucional de gran relevancia al momento de explicar la estabilidad de las bases del sistema de seguridad social chileno.
Un sistema de previsión no puede ser estático, supone una serie de adecuaciones en el tiempo a las distintas realidades demográficas, económicas, culturales y políticas. A diferencia de otros cambios, los procesos políticos en el área de la seguridad social, se mueven lentamente y van encadenados con sucesos anteriores. El análisis precedente no pretende desestimar la importancia o la necesidad de los cambios implementados al sistema. Se limita a afirmar que ellos no han afectado las bases del sistema y a atribuir al diseño constitucional y al rol del Tribunal Constitucional una integración al mismo de disposiciones novedosas que han permitido perfeccionar al sistema en armonía y con respeto a la concepción del hombre que ella plantea y los derechos fundamentales allí consagrados. Modificar de manera radical el sistema implicaría también la necesidad de reformar las bases de la institucionalidad misma de la Constitución y la de suprimir o limitar fuertemente en su esencia muchas de las garantías que ella consagra. En este sentido, y coincidiendo con lo que propone al respecto Cortázar ${ }^{25}$ es posible afirmar que el sistema de pensiones chileno, establecido con la lógica de capitalización individual otorga una protección relativa -aunque no absoluta- mayor que la del sistema de reparto a las presiones políticas, ya sean de naturaleza fiscal o redistributivas.
25 Cortázar, René (2003): Interacciones entre el Sistema de Pensiones y el Sistema Político (Seminario Federación de AFPs "Resultados y Desafíos de las Reformas a las Pensiones” México, Mayo 2003) pp. 2-4. 